\title{
Comment on 'A note on the formulation of the Maxwell equations for a macroscopic medium'
}

\author{
C. Vrejoiu and R. Zus \\ Department of Physics, University of Bucharest, PO Box MG-11, Bucharest, \\ Romania \\ E-mail : vrejoiu@fizica.unibuc.ro,roxana.zus@fizica.unibuc.ro
}

\begin{abstract}
.
In the present comment we emphasize the existence of a theorem on the equivalence of a polarized medium with an electric charged system in the dynamic case. This equivalence allows a consistent treatment of the polarization problem for a macroscopic medium.
\end{abstract}

In [1], writing the expressions for the scalar and vector potentials, expressions valid only in the case of statics,

$$
\Phi(\boldsymbol{r})=\frac{1}{4 \pi \varepsilon_{0}} \int \frac{\boldsymbol{P}\left(\boldsymbol{r}^{\prime}\right) \cdot\left(\boldsymbol{r}-\boldsymbol{r}^{\prime}\right)}{\left|\boldsymbol{r}-\boldsymbol{r}^{\prime}\right|^{3}} \mathbf{d}^{3} x^{\prime}=\frac{1}{4 \pi \varepsilon_{0}} \int \frac{-\nabla^{\prime} \cdot \boldsymbol{P}\left(\boldsymbol{r}^{\prime}\right)}{\left|\boldsymbol{r}-\boldsymbol{r}^{\prime}\right|} \mathbf{d}^{3} x^{\prime},
$$

and

$$
\boldsymbol{A}(\boldsymbol{r})=\frac{\mu_{0}}{4 \pi} \int \frac{\boldsymbol{M}\left(\boldsymbol{r}^{\prime}\right) \times\left(\boldsymbol{r}-\boldsymbol{r}^{\prime}\right)}{\left|\boldsymbol{r}-\boldsymbol{r}^{\prime}\right|^{3}} \mathrm{~d}^{3} x^{\prime}=\frac{\mu_{0}}{4 \pi} \int \frac{\nabla^{\prime} \times \boldsymbol{M}\left(\boldsymbol{r}^{\prime}\right)}{\left|\boldsymbol{r}-\boldsymbol{r}^{\prime}\right|} \mathrm{d}^{3} x^{\prime},
$$

the authors note a puzzling question for students in an introductory class of electromagnetism regarding the understanding of the equivalence of a polarized medium with charge and current distributions in the dynamic case. As these equations are commented in [1] (equations (5) and (6) from [1]), the contributions of a polarized medium to the field are established considering the dipolar electric and magnetic moments of each volume element expressed in terms of the polarization intensities $\boldsymbol{P}$ and $\boldsymbol{M}$. These contributions are determined using the well known expressions of the electromagnetic potentials of point electric and magnetic dipoles in the static case.

In the following we point out the existence of corresponding dynamic equations for the equations (1) and (2).

1) Firstly, we write the potentials of the electric and magnetic dipoles identified from the expansions of the retarded potentials in the exterior of an electric charge distribution confined in a finite domain $\mathcal{D}_{0}$. The expansion of the scalar potential is given by

$$
\begin{aligned}
4 \pi \varepsilon_{0} \Phi(\boldsymbol{r}, t) & =\sum_{n \geq 0} \frac{(-1)^{n}}{n !} \partial_{i_{1}} \ldots \partial_{i_{n}}\left[\frac{p_{i_{1} \ldots i_{n}}\left(t_{0}\right)}{r}\right] \\
& =\frac{q}{r}-\nabla\left[\frac{\boldsymbol{p}\left(t_{0}\right)}{r}\right]+\frac{1}{2} \partial_{i} \partial_{j}\left[\frac{p_{i j}\left(t_{0}\right)}{r}\right]+\ldots
\end{aligned}
$$


where

$$
p_{i_{1} \ldots i_{n}}(t)=\int_{\mathcal{D}_{0}} x_{i_{1}}^{\prime} \ldots x_{i_{n}}^{\prime} \rho\left(\boldsymbol{r}^{\prime}, t\right) \mathbf{d}^{3} x^{\prime},
$$

is the $n$-th order electric multipole moment, $q$ is the total electric charge, $O \in \mathcal{D}_{0}$ is the origin of coordinate system and $t_{0}=t-r / c$. For the vector potential, one has:

$$
\begin{aligned}
& \frac{4 \pi}{\mu_{0}} A_{i}(\boldsymbol{r}, t)=\sum_{n \geq 0} \frac{(-1)^{n}}{n !} \partial_{i_{1}} \ldots \partial_{i_{n}}\left[\frac{\mu_{i_{1} \ldots i_{n} i}\left(t_{0}\right)}{r}\right], \\
& \mu_{i_{1} \ldots i_{n} i}(t)=\int_{\mathcal{D}_{0}} x_{i_{1}}^{\prime} \ldots x_{i_{n}}^{\prime} j_{i}\left(\boldsymbol{r}^{\prime}, t\right) \mathbf{d}^{3} x^{\prime} .
\end{aligned}
$$

As it is known, [2, 3], using the equation

$$
j_{i}\left(\boldsymbol{r}^{\prime}, t\right)=\nabla^{\prime}\left(x_{i}^{\prime} \boldsymbol{j}\right)+x_{i}^{\prime} \partial \rho / \partial t
$$

resulting from the continuity equation $\partial \rho / \partial t+\nabla \cdot \boldsymbol{j}=0$, one can write for the contribution to the lowest order term in $A_{i}$ :

$\mu_{i}(t)=\int_{\mathcal{D}_{0}}\left[\nabla^{\prime}\left(x_{i}^{\prime} \boldsymbol{j}\right)+x_{i}^{\prime} \frac{\partial \rho}{\partial t}\right] \mathbf{d}^{3} x^{\prime}=\oint_{\mathcal{D}_{0}} x_{i}^{\prime} \boldsymbol{j} \cdot \mathbf{d} \boldsymbol{S}^{\prime}+\frac{\mathbf{d}}{\mathbf{d} t} \int_{\mathcal{D}_{0}} x_{i}^{\prime} \rho\left(\boldsymbol{r}^{\prime}, t\right) \mathbf{d}^{3} x^{\prime}=\dot{p}_{i}(t)$,

with the density $\boldsymbol{j}$ vanishing on the surface $\partial \mathcal{D}_{0}$ and $p_{i}(t)$ identified from equation (3). The $n=1$ term in the $A_{i}$ expansion is given by $-\partial_{k}\left(\mu_{k i} / r\right)$, where

$$
\begin{aligned}
\mu_{k i} & =\int_{\mathcal{D}_{0}} x_{k}^{\prime} j_{i} \mathbf{d}^{3} x^{\prime}=\int_{\mathcal{D}_{0}}\left[x_{k}^{\prime} \nabla^{\prime} \cdot\left(x_{i}^{\prime} \boldsymbol{j}\right)+x_{k}^{\prime} x_{i}^{\prime} \frac{\partial \rho}{\partial t}\right] \mathbf{d}^{3} x^{\prime} \\
& =\int_{\mathcal{D}_{0}} \nabla^{\prime} \cdot\left(x_{k}^{\prime} x_{i}^{\prime} \boldsymbol{j}\right) \mathbf{d}^{3} x^{\prime}-\int_{\mathcal{D}_{0}} x_{i}^{\prime} \boldsymbol{j} \cdot \nabla^{\prime} x_{k}^{\prime} \mathbf{d}^{3} x^{\prime}+\int_{\mathcal{D}_{0}} x_{k}^{\prime} x_{i}^{\prime} \frac{\partial \rho}{\partial t} \mathbf{d}^{3} x^{\prime}=-\int_{\mathcal{D}_{0}} x_{i}^{\prime} j_{k} \mathbf{d}^{3} x^{\prime}+\dot{p}_{k i} \\
& =-\int_{\mathcal{D}_{0}}\left(x_{i}^{\prime} j_{k}-x_{k}^{\prime} j_{i}\right) \mathbf{d}^{3} x^{\prime}-\int_{\mathcal{D}_{0}} x_{k}^{\prime} j_{i} \mathbf{d}^{3} x^{\prime}+\dot{p}_{k i}=-\varepsilon_{i k l} \int_{\mathcal{D}_{0}}\left(\boldsymbol{r}^{\prime} \times \boldsymbol{j}\right)_{l} \mathbf{d}^{3} x^{\prime}-\mu_{k i}+\dot{p}_{k i} .
\end{aligned}
$$

In the above calculation the relation $\nabla^{\prime} x_{k}^{\prime}=\boldsymbol{e}_{k}$ is considered, $\boldsymbol{e}_{k}$ being the orthogonal unit vectors along the cartesian axes. The integral of $\boldsymbol{\nabla}^{\prime}\left(x_{k}^{\prime} x_{i}^{\prime} \boldsymbol{j}\right)$ is zero because $\boldsymbol{j}=0$ on the surface $\partial \mathcal{D}_{0}$. Therefore, we can write

$$
\mu_{k i}(t)=-\varepsilon_{i k l} m_{l}(t)+\frac{1}{2} \dot{p}_{k i}(t)
$$

where

$$
\boldsymbol{m}(t)=\frac{1}{2} \int_{\mathcal{D}_{0}} \boldsymbol{r}^{\prime} \times \boldsymbol{j}\left(\boldsymbol{r}^{\prime}, t\right) \mathbf{d}^{3} x^{\prime}
$$

is the standard expression of the dipolar magnetic moment. Inserting the equations (4) and (5) in the expression of $\boldsymbol{A}$, we obtain

$$
\frac{4 \pi}{\mu_{0}} \boldsymbol{A}(\boldsymbol{r}, t)=\frac{\dot{\boldsymbol{p}}\left(t_{0}\right)}{r}+\boldsymbol{\nabla} \times\left[\frac{\boldsymbol{m}\left(t_{0}\right)}{r}\right]-\frac{1}{2} \boldsymbol{e}_{i} \partial_{k}\left[\frac{\dot{p}_{k i}\left(t_{0}\right)}{r}\right]+\ldots .
$$

The first two terms in the right-hand side of equation (6) represent the dipolar electric and magnetic contributions to the vector potential. From this result it is obvious that, 
together with the dipolar magnetic moment $\boldsymbol{m}$, the contribution of the 4-polar electric moment of the given distribution must be considered $[2,3]$. The general expression in terms of cartesian tensors for the expansion (6) is given in [4].

It is easy to identify the separate electric and magnetic dipolar contributions to the electromagnetic potentials and so, the potentials of the point electric and magnetic dipoles placed in the origin $O$ of the coordinate system:

$\Phi_{\boldsymbol{p}}(\boldsymbol{r}, t)=-\frac{1}{4 \pi \varepsilon_{0}} \boldsymbol{\nabla} \cdot\left[\frac{\boldsymbol{p}\left(t_{0}\right)}{r}\right]=\frac{1}{4 \pi \varepsilon_{0}}\left[\frac{\boldsymbol{r} \cdot \boldsymbol{p}\left(t_{0}\right)}{r^{3}}+\frac{\boldsymbol{r} \cdot \dot{\boldsymbol{p}}\left(t_{0}\right)}{c r^{2}}\right], \quad \boldsymbol{A}_{\boldsymbol{p}}(\boldsymbol{r}, t)=\frac{\mu_{0}}{4 \pi} \frac{\dot{\boldsymbol{p}}\left(t_{0}\right)}{r}$
$\Phi_{\boldsymbol{m}}(\boldsymbol{r}, t)=0, \quad \boldsymbol{A}_{\boldsymbol{m}}(\boldsymbol{r}, t)=\frac{\mu_{0}}{4 \pi} \boldsymbol{\nabla} \times\left[\frac{\boldsymbol{m}\left(t_{0}\right)}{r}\right]=\frac{\mu_{0}}{4 \pi}\left[\frac{\boldsymbol{m}\left(t_{0}\right) \times \boldsymbol{r}}{r^{3}}+\frac{\dot{\boldsymbol{m}}\left(t_{0}\right) \times \boldsymbol{r}}{c r^{2}}\right]$.

2) In the point given by the vector $\boldsymbol{r}$, the contribution to the field of the polarized medium localized in a domain $\mathcal{D}$ is obtained as a sum over all electric and magnetic dipolar contributions of this medium: each volume element $\mathbf{d} V$ contributes with the electric dipolar moment $\boldsymbol{P} \mathbf{d} V$ and a magnetic one $\boldsymbol{M} \mathbf{d} V$. The potentials are given by

$$
\Phi(\boldsymbol{r}, t)=-\frac{1}{4 \pi \varepsilon_{0}} \int_{\mathcal{D}} \nabla \frac{\boldsymbol{P}\left(\boldsymbol{r}^{\prime}, \tau\right)}{R} \mathbf{d}^{3} x^{\prime}
$$

and

$$
\boldsymbol{A}(\boldsymbol{r}, t)=\frac{\mu_{0}}{4 \pi} \int_{\mathcal{D}} \boldsymbol{\nabla} \times \frac{\boldsymbol{M}\left(\boldsymbol{r}^{\prime}, \tau\right)}{R} \mathbf{d}^{3} x^{\prime}+\frac{\mu_{0}}{4 \pi} \int_{\mathcal{D}} \frac{\dot{\boldsymbol{P}}\left(\boldsymbol{r}^{\prime}, \tau\right)}{R} \mathbf{d}^{3} x^{\prime},
$$

where $\boldsymbol{R}=\boldsymbol{r}-\boldsymbol{r}^{\prime}$ and $\tau=t-R / c$ is the retarded time as a function of $\boldsymbol{r}$ and $\boldsymbol{r}^{\prime}$. The static expressions corresponding to equations (7) and (8) are found in equations (1) and (2), respectively.

For simplicity, we consider all the properties varying continuously in the domain $\mathcal{D}$, but some parameters may present discontinuities across the boundary $\partial \mathcal{D}$.

The integrant in equation (7) can be expressed as

$$
\boldsymbol{\nabla} \frac{\boldsymbol{P}\left(\boldsymbol{r}^{\prime}, \tau\right)}{R}=\boldsymbol{P}\left(\boldsymbol{r}^{\prime}, \tau\right) \cdot \nabla \frac{1}{R}-\frac{\dot{\boldsymbol{P}}\left(\boldsymbol{r}^{\prime}, \tau\right) \cdot \nabla R}{c R}=-\boldsymbol{P}\left(\boldsymbol{r}^{\prime}, \tau\right) \cdot \nabla^{\prime} \frac{1}{R}+\frac{\dot{\boldsymbol{P}}\left(\boldsymbol{r}^{\prime}, \tau\right) \cdot \nabla^{\prime} R}{c R} .
$$

Using the notation $\left[\partial f\left(\boldsymbol{r}^{\prime}, t^{\prime}\right) / \partial x_{i}^{\prime}\right]_{t^{\prime}=\tau}$ for the retarded value of the derivative of $f\left(\boldsymbol{r}^{\prime}, t^{\prime}\right)$, we can write

$$
\left[\frac{\partial}{\partial x_{i}^{\prime}} f\left(\boldsymbol{r}^{\prime}, t^{\prime}\right)\right]_{t^{\prime}=\tau}=\frac{\partial}{\partial x_{i}^{\prime}} f\left(\boldsymbol{r}^{\prime}, \tau\right)+\frac{1}{c} \dot{f}\left(\boldsymbol{r}^{\prime}, \tau\right) \frac{\partial R}{\partial x_{i}^{\prime}},
$$

and, particularly,

$$
\frac{\dot{\boldsymbol{P}}\left(\boldsymbol{r}^{\prime}, \tau\right) \cdot \nabla^{\prime} R}{c R}=\frac{\left[\nabla^{\prime} \cdot \boldsymbol{P}\left(\boldsymbol{r}^{\prime}, t^{\prime}\right)\right]_{t^{\prime}=\tau}}{R}-\frac{\boldsymbol{\nabla}^{\prime} \cdot \boldsymbol{P}\left(\boldsymbol{r}^{\prime}, \tau\right)}{R} .
$$

Equation (9) becomes

$$
\begin{aligned}
\boldsymbol{\nabla} \frac{\boldsymbol{P}\left(\boldsymbol{r}^{\prime}, \tau\right)}{R} & =-\boldsymbol{P}\left(\boldsymbol{r}^{\prime}, \tau\right) \cdot \nabla^{\prime} \frac{1}{R}+\frac{\left[\boldsymbol{\nabla}^{\prime} \boldsymbol{P}\left(\boldsymbol{r}^{\prime}, t^{\prime}\right)\right]_{t^{\prime}=\tau}}{R}-\frac{\boldsymbol{\nabla}^{\prime} \cdot \boldsymbol{P}\left(\boldsymbol{r}^{\prime}, \tau\right)}{R} \\
& =\frac{\left[\boldsymbol{\nabla}^{\prime} \cdot \boldsymbol{P}\left(\boldsymbol{r}^{\prime}, t^{\prime}\right)\right]_{t^{\prime}=\tau}}{R}-\boldsymbol{\nabla}^{\prime} \frac{\boldsymbol{P}\left(\boldsymbol{r}^{\prime}, \tau\right)}{R}
\end{aligned}
$$


Using this result in equation (7), we obtain

$$
\Phi(\boldsymbol{r}, t)=\frac{1}{4 \pi \varepsilon_{0}} \int_{\mathcal{D}} \frac{-\left[\boldsymbol{\nabla}^{\prime} \cdot \boldsymbol{P}\left(\boldsymbol{r}^{\prime}, t^{\prime}\right)\right]_{t^{\prime}=\tau}}{R} \mathbf{d}^{3} x^{\prime}+\frac{1}{4 \pi \varepsilon_{0}} \oint_{\partial \mathcal{D}} \frac{\boldsymbol{P}\left(\boldsymbol{r}^{\prime}, \tau\right) \cdot \mathbf{d} \boldsymbol{S}^{\prime}}{R} .
$$

From the last equation, we can identify the expressions of the volume and surface macroscopic polarization charge densities:

$$
\rho_{\boldsymbol{P}}(\boldsymbol{r}, t)=-\boldsymbol{\nabla} \cdot \boldsymbol{P}(\boldsymbol{r}, t), \quad \sigma_{p}(\boldsymbol{r}, t)=-\boldsymbol{n} \cdot \boldsymbol{P}(\boldsymbol{r}, t),
$$

$\boldsymbol{n}$ being the normal on the boundary $\partial \mathcal{D}$ in the point $\boldsymbol{r}$.

In equation (8) we have

$$
\begin{aligned}
\nabla \times \frac{\boldsymbol{M}\left(\boldsymbol{r}^{\prime}, \tau\right)}{R} & =\left(\nabla \frac{1}{R}\right) \times \boldsymbol{M}\left(\boldsymbol{r}^{\prime}, \tau\right)+\frac{\boldsymbol{\nabla} \times \boldsymbol{M}\left(\boldsymbol{r}^{\prime}, \tau\right)}{R} \\
& =-\left(\boldsymbol{\nabla}^{\prime} \frac{1}{R}\right) \times \boldsymbol{M}\left(\boldsymbol{r}^{\prime}, \tau\right)+\frac{\left(\boldsymbol{\nabla}^{\prime} R\right) \times \dot{\boldsymbol{M}}\left(\boldsymbol{r}^{\prime}, \tau\right)}{c R} .
\end{aligned}
$$

Using equation (10) for expresing the term $\left(\nabla^{\prime} R\right) \dot{M}\left(\boldsymbol{r}^{\prime}, \tau\right) / c R$, we obtain $\ddagger$

$$
\nabla \times \frac{M\left(\boldsymbol{r}^{\prime}, \tau\right)}{R}=\frac{\left[\nabla^{\prime} \times \boldsymbol{M}\left(\boldsymbol{r}^{\prime}, t^{\prime}\right)\right]_{t^{\prime}=\tau}}{R}-\nabla^{\prime} \times \frac{\boldsymbol{M}\left(\boldsymbol{r}^{\prime}, \tau\right)}{R},
$$

such that the equation (8) can be written in the following form

$$
\begin{aligned}
\boldsymbol{A}(\boldsymbol{r}, t) & =\frac{\mu_{0}}{4 \pi}\left(\int_{\mathcal{D}} \frac{\left[\boldsymbol{\nabla}^{\prime} \times \boldsymbol{M}\left(\boldsymbol{r}^{\prime}, t^{\prime}\right)\right]_{t^{\prime}=\tau}}{R} \mathbf{d}^{3} x^{\prime}+\oint_{\partial \mathcal{D}} \frac{\boldsymbol{M}\left(\boldsymbol{r}^{\prime}, \tau\right) \times \boldsymbol{n}}{R} \mathrm{~d} S^{\prime}\right) \\
& +\frac{\mu_{0}}{4 \pi} \int_{\mathcal{D}} \frac{\dot{\boldsymbol{P}}\left(\boldsymbol{r}^{\prime}, \tau\right)}{R} \mathbf{d}^{3} x^{\prime} .
\end{aligned}
$$

We can identify the expressions of the volume macroscopic polarization current densities:

$$
\boldsymbol{j}_{p o l}=\boldsymbol{j}_{M}+\boldsymbol{j}_{\boldsymbol{P}}: \quad \boldsymbol{j}_{\boldsymbol{M}}(\boldsymbol{r}, t)=\nabla \times \boldsymbol{M}(\boldsymbol{r}, t), \quad \boldsymbol{j}_{\boldsymbol{P}}=\frac{\partial \boldsymbol{P}(\boldsymbol{r}, t)}{\partial t},
$$

and the surface polarization current density

$$
\boldsymbol{k}_{\boldsymbol{M}}(\boldsymbol{r}, t)=\boldsymbol{M}(\boldsymbol{r}, t) \times \boldsymbol{n} .
$$

No matter how the macroscopic equations are introduced, their interpretation as Maxwell equations in vacuum for the fields $\boldsymbol{E}$ and $\boldsymbol{B}$ associated with the bound charge and current distributions (12) and (14), together with the "free" charges and currents $\rho$ and $\boldsymbol{j}$ is a direct consequence of these equations.

We point out that the mathematical identity which corresponds to equation (2) in the dynamic case is the following $\S$

$$
\int \frac{\left[\nabla^{\prime} \times M\left(\boldsymbol{r}^{\prime}, t^{\prime}\right)\right]_{t^{\prime}=\tau}}{R} \mathbf{d}^{3} x^{\prime}=\int \nabla \times \frac{M(\boldsymbol{r}, \tau)}{R} \mathbf{d}^{3} x^{\prime}
$$

The relation implied in obtaining the result (13) is, in fact, the equation (16) with the integral $\int \dot{\boldsymbol{P}}\left(\boldsymbol{r}^{\prime}, \tau\right) / R \mathbf{d}^{3} x^{\prime}$ added on both hand sides. Adding this term to equation

$\ddagger$ For expressing the curl operator using equation (10), it is usefull writing $\boldsymbol{\nabla} \times \boldsymbol{V}=\boldsymbol{e}_{i} \varepsilon_{i j k} \partial_{j} V_{k}$.

$\S$ We thank an unknown referee for helping us to clarify this point. 
(16) corresponds to the correct introduction of the distributions of polarization charge and currents $\rho_{\boldsymbol{P}}$ and $\boldsymbol{j}_{\text {pol }}$, the part represented by $\dot{\boldsymbol{P}}$ ensuring the continuity equation $\partial \rho_{\boldsymbol{P}} / \partial t+\boldsymbol{\nabla} \cdot \boldsymbol{j}_{\text {pol }}=0$. The term $\dot{\boldsymbol{P}}$ added to the polarization current represents the medium polarization part of the Maxwell displacement current. In conclusion, starting from the polarization charge and current distributions for proving the two possible representations of the polarized medium contributions to the field, the trick of adding the term $\dot{\boldsymbol{P}}$ corresponds to the Maxwell's hypothesis regarding the displacement current. Note that the same artifice has to be imposed when requiring that potentials fulfil the Lorenz condition. The equivalance of the two methods is obvious if one keeps in mind that the retarded potentials fulfil the Lorenz condition, as a consequence of the electric charge continuity equation.

Finally, we point out that given the dipolar electric and magnetic distributions by the domain functions

$$
\mathcal{D} \longrightarrow \boldsymbol{p}(\mathcal{D}, t)=\int_{\mathcal{D}} \boldsymbol{P}(\boldsymbol{r}, t) \mathbf{d}^{3} x^{\prime}, \quad \mathcal{D} \longrightarrow \boldsymbol{m}(\mathcal{D}, t)=\int_{\mathcal{D}} \boldsymbol{M}(\boldsymbol{r}, t) \mathbf{d}^{3} x^{\prime},
$$

these distributions are equivalent with the charge and current distributions given by equations (14) and (15).

3) The results from items 1) and 2) are useful for understanding the microscopic nature of the macroscopic electric and magnetic polarizations of a medium. A complex microscopic system, as a molecule for example, may be represented as a confined electric charged system in a domain $\mathcal{D}_{a}$ centered on a point $P_{a}\left(\boldsymbol{r}_{a}\right)$. Let the averaging process be used for obtaining the macroscopic parameters of a medium. The molecules contribute by the multipole electric and magnetic moments which appear in the multipole expansions of their microscopic fields. In a simple model with electrical neutral molecules and $\boldsymbol{r}_{a}$ time independent, the microscopic densities are represented by the multipole series [2]

$$
\rho_{\text {micro }}=\sum_{a} \rho^{(a)}, \quad \boldsymbol{j}_{\text {micro }}=\sum_{a} \boldsymbol{j}^{(a)}
$$

where $[2,4]$

$$
\rho^{(a)}(\boldsymbol{r}, t)=-\partial_{i}\left\{\left[p_{i}^{(a)}(t)-\frac{1}{2} p_{i k}^{(a)}(t) \partial_{k}+\frac{1}{6} p_{i j k}^{(a)}(t) \partial_{j} \partial_{k}+\ldots\right] \delta\left(\boldsymbol{r}-\boldsymbol{r}_{a}\right)\right\}
$$

and

$$
\begin{aligned}
j_{i}^{(a)}(\boldsymbol{r}, t) & =\varepsilon_{i k l} \partial_{k}\left\{\left[m_{l}^{(a)}(t)-\frac{1}{2} m_{q k}^{(a)}(t) \partial_{q}+\ldots\right] \delta\left(\boldsymbol{r}-\boldsymbol{r}_{a}\right)\right\} \\
& +\frac{\partial}{\partial t}\left\{\left[p_{i}^{(a)}(t)-\frac{1}{2} p_{i k}^{(a)}(t) \partial_{k}+\frac{1}{6} p_{i j k}^{(a)}(t) \partial_{j} \partial_{k} \ldots\right] \delta\left(\boldsymbol{r}-\boldsymbol{r}_{a}\right)\right\} .
\end{aligned}
$$

In equations (17) and (18) the series expansions are given up to the 8-polar electric terms and 4-polar magnetic ones $\|$. The electric and magnetic moments of a molecule indexed \| The 4-polar magnetic moment is defined by the components $m_{i k}=\frac{2}{3} \int_{\mathcal{D}_{0}} x_{i}\left(\boldsymbol{r}^{\prime} \times \boldsymbol{j}\right)_{k} \mathbf{d}^{3} x^{\prime}$. 
by $a$ are represented by the tensor components $p_{i}, p_{i j}, p_{i j k}$ and $m_{i}, m_{i j}$, respectively. The general terms of the series are given in literature [4] $\uparrow$.

The microscopic densities $\rho_{\text {micro }}$ and $\boldsymbol{j}_{\text {micro }}$ can be written as

$$
\rho_{\text {micro }}=-\boldsymbol{\nabla} \cdot \boldsymbol{P}_{\text {micro }}, \boldsymbol{j}_{\text {micro }}=\boldsymbol{\nabla} \times \boldsymbol{M}_{\text {micro }}+\frac{\partial}{\partial t} \boldsymbol{P}_{\text {micro }},
$$

where $\boldsymbol{P}_{\text {micro }}$ and $\boldsymbol{M}_{\text {micro }}$ are sums over all molecules and all orders of molecular polarization moments resulting from equations (17) and (18). Therefore, the equations (12) and (14) for the volume densities are obtained from the averaged equations (19) with $\boldsymbol{P}=\left\langle\boldsymbol{P}_{\text {micro }}\right\rangle$, and $\boldsymbol{M}=\left\langle\boldsymbol{M}_{\text {micro }}\right\rangle$. Finally, from equations (17), (18) and (19) it is obvious how the macroscopic dipolar polarization of a medium is generated by all orders molecular multipoles.

Starting from the microscopic field equations, the part $\dot{\boldsymbol{P}}$ of the macroscopic displacement current is a consequence of the Maxwell-Lorentz equations and of the multipole expansions of the microscopic charge and current distributions.

If a class of electrodynamics begins from the microscopic Maxwell-Lorentz equations, then the student can understand some subtleties of the averaging procedure used for obtaining the macroscopic Maxwell equations.

\section{References}

[1] Leung P T, Ni G J 2008 Eur. J. Phys. 29, N37-N41

[2] Jackson J. D. Classical Electrodynamics- third edition (Wiley New York, 1998)

[3] Raab R. E., De Lange O. L. Multipole Theory in Electromagnetism (Clarendon Press Oxford, 2005)

[4] Vrejoiu C 2002 J. Phys. A: Math. Gen. 35, 9911-22

I Because the magnetism is inexplicable in classical physics (the Van Leeuwen theorem), in the above expressions of microscopic densities, the quantum averaged parameters must be considered. 